\title{
Lidil
}

Revue de linguistique et de didactique des langues

$37 \mid 2008$

Syntaxe et sémantique des prédicats

\section{Entre événements et actions}

Les schèmes composés de constructions syntaxiques du dictionnaire Les verbes Français

de J. Dubois \& F. Dubois-Charlier

Jacques François

\section{OpenEdition}

\section{Journals}

Édition électronique

URL : http://journals.openedition.org/lidil/2703

DOI : $10.4000 /$ lidil.2703

ISSN : 1960-6052

\section{Éditeur}

UGA Éditions/Université Grenoble Alpes

\section{Édition imprimée}

Date de publication : 1 juin 2008

Pagination : 175-190

ISBN : 978-2-84310-124-3

ISSN : $1146-6480$

Référence électronique

Jacques François, « Entre événements et actions », Lidil [En ligne], 37 | 2008, mis en ligne le 01 septembre 2009, consulté le 02 mai 2019. URL : http://journals.openedition.org/lidil/2703 ; DOI 10.4000/lidil.2703

(C) Lidil 


\title{
ENTRE ÉVÉNEMENTS ET ACTIONS
}

\author{
LES SCHÈMES COMPOSÉS DE CONSTRUCTIONS \\ SYNTAXIQUES DU DICTIONNAIRE LES VERBES FRANÇAIS \\ DE J. DuboIS \& F. DubOIS-CHARLIER
}

Jacques FRANÇOIS*

\begin{abstract}
RÉSUMÉ
Dans le dictionnaire Les Verbes français (1997), J.Dubois et F. Dubois-Charlier proposent un classement primairement distributionnel et transformationnel et secondairement sémantique de 12310 verbes français représentant plus de 25000 entrées. Certains schèmes syntaxiques associés aux entrées comportent une composante transitive directe, pronominale, transitive indirecte et/ou intransitive sur la base de relations transformationnelles répertoriées. L'orientation de la relation est pertinente et à côté de 8891 schèmes composés du type $\mathrm{T} \rightarrow \mathrm{P}$, on rencontre également 1264 schèmes inverses du type $\mathrm{P} \rightarrow \mathrm{T}$, contrairement à la conception des diathèses réfléchie, réciproque et récessive de Tesnière. La notion de «transitivité (non) factitive » permet partiellement de saisir la raison d'être du type $P \rightarrow T$, mais certains de ses soustypes restent sujets à caution.
\end{abstract}

\section{ABSTRACT}

In the dictionary Les Verbes français (1997), J.Dubois \& $F$. Dubois-Charlier set out a classification of 12310 French verbs amounting to more than 25.000 lexical entries on a distributional and transformational basis, but with a semantic ouput. This article aims at identifying the criteria positing a type $P$ (ronominal) $\rightarrow$ $T$ (ransitive) of composed syntactic schemes beside the classical converse type $T \rightarrow P$ as expounded by $L$. Tesnière in his theory of verb diatheses. The distinction between factitive and non factitive transitivity yields a partial justification of the $P \rightarrow T$ type, but some of its subtypes remain questionable.

*Université de Caen, CRISCo (Fre 2805, CNRS) 
Cet article ${ }^{1} \mathrm{~s}^{\prime}$ inscrit dans le prolongement du numéro $153 \mathrm{de}$ Langue Française, que j'ai coordonné avec Denis Le Pesant et Danielle Leeman. Ce numéro paru en mars 2007 est consacré à l'examen des classes syntactico-sémantiques et de la polysémie verbale dans le dictionnaire Les verbes français [désormais abrégé $L V F$ ] de Jean Dubois et Françoise DuboisCharlier [abrégés JD \& FDC], qui a été diffusé par Larousse en 1997 et dont la version électronique source doit être prochainement disponible sur le site de 1'équipe MoDyCo (Université Paris 10 \& CNRS). Je renvoie particulièrement à la présentation générale des différents niveaux de classement hiérarchique des constructions verbales selon JD \& FDC (François, Le Pesant \& Leeman, 2007).

L'objet de l'article est l'examen d'une propriété du classement de $L V F$ qui n'a pu être abordée que superficiellement dans l'article introductif mentionné ci-dessus: aux entrées lexicales de ce dictionnaire est très fréquemment associé un «schème de construction syntaxique» composé de plusieurs « variantes issues des diverses modifications ou transformations de la phrase et formant un ensemble de phrases reliées les unes aux autres ${ }^{2} »$. Parmi ces variantes, les auteurs mentionnent:

- objet direct animé devenant sujet d'un pronominal réfléchi (on a fourré Paul dans une sale histoire, Paul s'est fourré dans une sale histoire)

La formulation évoque soit la «diathèse réfléchie », si Paul est supposé responsable de ce qui lui arrive, soit dans le cas inverse la «diathèse récessive à marquant réfléchi » de Tesnière $(1969$, chap. 115, p. $272 s q)$. On est donc conduit à supposer que dans une interprétation comme dans l'autre, dans le cas d'une relation transformationnelle entre une structure transitive et une structure pronominale, la structure transitive est toujours (comme chez Tesnière) à la source du processus,

1. Je remercie I. Novakova pour ses commentaires judicieux sur l'affinité entre les diathèses évoquées ici et celles que manifestent les langues slaves, en particulier le russe et le bulgare.

2. $L V F$, p. IV, soulignement des auteurs, repris dans Dubois \& Dubois-Charlier 1997b, p. 53. 
la structure pronominale en étant l'aboutissement $(\mathrm{T} \rightarrow \mathrm{P})$. Or l'examen de l'édition de 1997 nous met en présence de schèmes composés inverses où la structure pronominale est la source et la structure transitive le terme du processus transformationnel $(\mathrm{P} \rightarrow \mathrm{T})$. La consultation de la base de données électronique ${ }^{3}$ confirme cette constatation et permet de dégager deux ensembles de relations transformationnelles $\mathrm{T} \rightarrow \mathrm{P}$ et $\mathrm{P} \rightarrow \mathrm{T}$ qui sont largement différenciées mais qui toutefois se superposent occasionnellement, c'est-à-dire que - pour des propriétés identiques du sujet, de l'objet et d'autres constituants conventionnellement au nombre de 4 que je noterai T...• et Po..• in abstracto - certaines relations transformation-

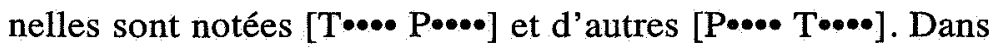
le premier cas la structure transitive est la source de la transformation et la structure pronominale le terme, dans le second cas c'est l'inverse. La question qui est posée ici est donc celle des CRITÈRES - manifestement d'ordre sémantique - D'ORIENTATION DE LA RELATION TRANSFORMATIONNELLE qui met en jeu d'un côté une représentation d'action, de l'autre soit une représentation d'évènement ${ }^{4}$ (par exemple La cruche se casse $\rightarrow$ La servante a cassé la cruche), soit une représentation d'action réfléchie (par exemple Paul s'est rasé $\rightarrow$ Le barbier a rasé $P a u l$ ), soit enfin une représentation modale ou générique d'action ${ }^{5}$ (par exemple Les cruches en terre cuite se cassent facilement $\rightarrow$ On casse facilement les cruches en terre cuite).

Les "schèmes de construction syntaxique ", maillon central de la hiérarchisation syntactico-sémantique du dictionnaire Les verbes français

La démarche de JF \& FDC s'articule en trois temps ${ }^{6}$ :

3. Je remercie J. Dubois et $F$. Dubois-Charlier de m'avoir laissé consulter cette base de données avant qu'elle ne soit accessible sur le site de MoDyCo.

4. Construction «moyenne» selon Ruwet (1972).

5. Construction «neutre» selon la même source.

6. Pour une présentation détaillée, voir François, Le Pesant \& Leeman (2007, \& 1.4: La hiérarchie des classes). 
i. un premier temps de collecte empirique d'un maximum de contextes pour un maximum de verbes français et de distinctions d'entrées lexicales (jusqu'à 61 pour le verbe passer);

ii. un second temps de rassemblement hiérarchique progressif «de bas en haut» des entrées jusqu'à un petit nombre de classes dites «génériques », c'est-à-dire conceptuelles, en attribuant à chaque entrée un profil sémantique appelé «opérateur», lui-même composé d'une combinaison de primitives sémantiques ${ }^{7}$; sur cette base il a été possible de constituer la base de données électronique et la section Index de l'ouvrage de 1997 établissant pour chaque verbe l'éventail de ses entrées, lequel constitue indirectement son champ sémasiologique par l'intermédiaire du jeu des opérateurs associés à chaque entrée;

iii. un troisième temps d'inversion de la perspective et de composition de la section Les classes de verbe de l'ouvrage édité, laquelle procède de haut en bas à partir des 14 classes génériques.

A partir du repérage des contextes d'emploi et de la distinction des entrées, une multitude de schèmes de construction syntaxique est répertoriée. Certains schèmes se rassemblent en schèmes composés. Schèmes élémentaires et schèmes composés sont rassemblés en sous-types, eux-mêmes regroupés progressivement en 248 sous-classes syntaxiques, 54 classes sémantico-syntaxiques et finalement 14 classes génériques. L'Index de $L V F$ attribue à chaque entrée, par exemple bouger 01, une description à trois niveaux, en l'occurrence la classe générique ' $M$ ' pour « faire ou imprimer tel mouvement», la classe sémantico-syntaxique ' 3 ' pour «sujet/objet non-animé, et la sous-classe syntaxique 'a' pour «faire mouvement». Le verbe bouger présente 9 entrées plus ou moins apparentées les unes aux autres sur la base de leurs descriptions à trois niveaux. Sur le tableau 1 , les entrées sont

7. Ces primitives s'apparentent à celles que l'équipe d'Anna Wierzbicka continue inlassablement de cataloguer, cf. Peeters (dir.2006) pour le plaidoyer le plus récent pour une «métalangue sémantique naturelle» et son application à quatre langues romanes. 
disposées dans l'ordre de leurs descriptions. Cinq entrées sont de la classe générique $M$ (mouvement), dont deux de la classe sémantico-syntaxique 3 , deux sont de la classe générique $\mathrm{E}$, une de la classe $C$ et une de la classe $T$.

\begin{tabular}{|c|c|c|c|c|}
\hline $\begin{array}{l}\text { Classe } \\
\text { générique }\end{array}$ & $\begin{array}{l}\text { Classe } \\
\text { sémantico } \\
\text { syntaxique }\end{array}$ & $\begin{array}{l}\text { Sous-classe } \\
\text { syntaxique }\end{array}$ & Entrée & nple \\
\hline \multirow[t]{3}{*}{$\begin{array}{l}\mathrm{C} \text { (com- } \\
\text { munication) }\end{array}$} & 1 (s'exprimer) & $\begin{array}{l}f \text { (discours sur/ } \\
\text { contre) }\end{array}$ & bouger 05 & $\begin{array}{l}\text { Les jour } \\
\text { naux } \\
\text { n'ont } \\
\text { pas bougé }\end{array}$ \\
\hline & 1 (suj hum) & a (sortir de qp) & bouger 03 (s) & $\begin{array}{l}\text { Bouge-toi } \\
\text { de là! }\end{array}$ \\
\hline & 3 (suj non-anim) & $\begin{array}{l}c \text { (faire sortir } \\
\text { qc de lieu) }\end{array}$ & bouger 04 & $\begin{array}{l}\text { On a } \\
\text { bougé mes }\end{array}$ \\
\hline \multirow[t]{3}{*}{$\begin{array}{l}\text { E (entrer/ } \\
\text { sortir) }\end{array}$} & & & & $\begin{array}{l}\text { papiers } d u \\
\text { bureau }\end{array}$ \\
\hline & 1 (suj hum, non figuré) & a (faire mvt) & bouger 02 & $\begin{array}{l}\text { Paul } \\
\text { bouge }\end{array}$ \\
\hline & 2 (suj hum, figure) & $\begin{array}{l}\text { a (faire mvt en } \\
\text { pensée, } \\
\text { atteindre }\end{array}$ & & $\begin{array}{l}\text { sur sa } \\
\text { chaise }\end{array}$ \\
\hline \multirow[t]{4}{*}{$\begin{array}{l}\text { M (taire/ } \\
\text { impri- } \\
\text { mer mvt) }\end{array}$} & & $\begin{array}{l}\text { dévelop- } \\
\text { pement) }\end{array}$ & bouger 06 & $\begin{array}{l}\text { Çabouge } \\
\text { en } \\
\text { Amérique } \\
\text { centrale }\end{array}$ \\
\hline & $\begin{array}{l}3 \text { (sujet non animé, } \\
\text { non figuré) }\end{array}$ & $a($ faire $m v t)$ & bouger 01 & $\begin{array}{l}\text { La dent } \\
\text { bouge }\end{array}$ \\
\hline & & $\mathrm{b}$ (imprimer $\mathrm{mvt}$ ) & bouger 09 & $\begin{array}{l}\text { Marie } \\
\text { bouge } \\
\text { le bras }\end{array}$ \\
\hline & $\begin{array}{l}4 \text { (sujet non anime, } \\
\text { figure) }\end{array}$ & $\begin{array}{l}\text { a (faire mvt bouger } 08 \\
\text { abstr) }\end{array}$ & $\begin{array}{l}\text { Les salaires ne bougent } \\
\text { pas }\end{array}$ & \\
\hline $\mathrm{T}$ (changer) & $\begin{array}{l}4 \text { (sujet non animé, } \\
\text { figure) }\end{array}$ & $\begin{array}{l}\mathrm{d} \text { (se trans- bouger } 07 \\
\text { former } \\
\text { en qc) }\end{array}$ & $\begin{array}{l}\text { Les couleurs ne } \\
\text { bougent pas }\end{array}$ & \\
\hline
\end{tabular}


Dans la démarche d'élaboration de la section «Classes de verbe» de la version éditée, la présentation hiérarchique est inversée : chaque classe générique ${ }^{8}$ se décompose en plusieurs (en général 4) classes sémantico-syntaxiques, celles-ci en un nombre variable de sous-classes syntaxiques, lesquelles donnent lieu à leur tour à différents sous-types qui rassemblent différents schèmes élémentaires ou composés. Ceux-ci peuvent regrouper occasionnellement plusieurs centaines d'entrées. Sur la figure 1, la partie du bas reprend les trois niveaux hiérarchiques majeurs mentionnés dans le tableau 1. La partie du haut montre qu'à l'entrée bouger 03 (s) est associé un schème [A13 P1030] composé de deux schèmes élémentaires qui spécifient chacun un type de construction tran-

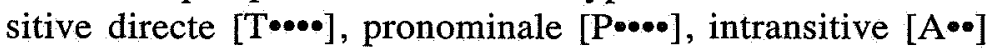
ou transitive indirecte $[\mathrm{N} \bullet \cdot]$, chaque $\langle\bullet>$ symbolisant une valeur de propriété du sujet grammatical, de l'objet direct, de la préposition introduisant un objet indirect, etc. L'opérateur sémantique ex. D lc qp (SORTIR DE LIEU QUELQUE PART) s'applique au schème composé: que le verbe soit employé en construction intransitive ou pronominale est sémantiquement neutre pour cette entrée.

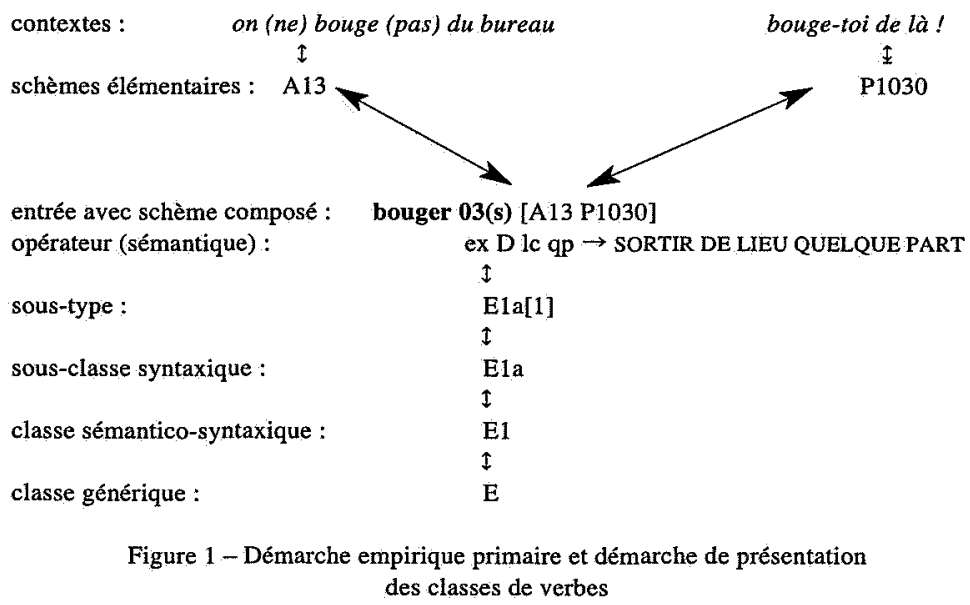

8. À l'exception de la dernière classe générique qui a une raison d'être fonctionnelle et non sémantique: c'est celle des auxiliaires et semi-auxiliaires. 


\section{Les schèmes composés à priorité transitive (T....-P.....) ou pronominale (P.....-T.....)}

Sur les 25609 entrées du dictionnaire $L V F, 8891$, soit $35 \%$ sont associées à un schème syntaxique composé d'au moins T*.* en position primaire et P.... en position secondaire. Le type de construction intransitive [A*•] peut également être représenté, par exemple

bloquer 02 (SYN: immobiliser)

S3i [T1308 A30 P3000]

On bloque les roues, un volet. La porte, la clef se bloque. Le verrou bloque.

Inversement, 1264 entrées présentent un schème composé inversé avec P...• en position primaire et T...• en position secondaire. Ici, l'un ou l'autre des deux autres types de construction peut également figurer, par exemple

angoisser (s) (SYN: s'inquiéter)

P1a [P1000 A10 T3100]

On angoisse, s'angoisse, est angoissé de le voir. Cette prédiction angoisse

Paul.

À priori la justification de l'orientation de la relation transformationnelle est passablement mystérieuse, d'autant plus que l'introduction de l'ouvrage, reprise littéralement dans l'article de 1997, n'entre dans aucun détail. À l'appui de cette observation, quelques illustrations (1-5, l'ordre des exemples ne fournit pas d'indice):

(1) Paul s'amuse avec des riens. Un rien amuse Paul. On amuse Paul avec un rien.

(2) On case les valises dans le coffre. Le sac se case dans le coffre.

(3) On sectionne le câble. La machine a sectionné le bras à $P$. Le câble s'est sectionné.

(4) La blessure s'irrite. Cette crème irrite la plaie.

(5) Marie s'apprête pour la soirée. Marie apprête les enfants pour l'école.

Le schème composé associé à (1), entrée amuser 01(s), est P1000 (sujet hum) T1108 (sujet et objet hum + compl. instrumental), c'est-à-dire que la construction pronominale est considérée comme primaire, sans doute parce que Paul assume un rôle d'expérient. Il en est de même pour (4), entrée irriter 02(s) avec le schème P3006 (sujet non animé, compl. de modalité) T3300 (sujet et objet non animés), ce qui parait 
logique, car la blessure subit l'irritation occasionnée par la crème. Mais aussi pour (5), entrée apprêter 02(s) avec le schème P1000 (sujet animé) T1100 (sujet et objet animés). Or dans ce cas, Marie accomplit bien une action sur elle-même (elle apprête sa coiffure, sa tenue, son maquillage, etc.). II parait donc étonnant que la construction pronominale - qui ne peut pas exprimer une diathèse récessive, dans la terminologie de Tesnière - puisse être primaire. Inversement (2), l'entrée caser 01 se voit attribuer le schème T13j0 (sujet hum, objet non animé, compl. en/dans) P30j0 (sujet non animé, même compl.). De la faible acceptabilité de La valise s'est casée dans le coffre, à moins d'ajouter facilement/difficilement, on déduit que la construction pronominale a ici une valeur générique ou modale. Une telle valeur est manifestement dérivée, il est donc logique que la construction transitive soit primaire. Mais en (3), l'entrée sectionner 01 est associée au schème T13j8 (sujet hum, objet non animé + compl. en/dans + compl. instrumental) P30j0 (sujet hum + compl. en/dans), ce qui indique que dans l'esprit des auteurs l'irritation de la plaie est imaginable indépendamment d'une instance causatrice particulière, alors que ce n'est pas le cas pour le sectionnement du câble. Mais on peut imaginer un contexte dans lequel on observe que le câble est sectionné sans qu'on sache ce qui s'est passé, auquel cas il faut bien s'imaginer sa rupture par elle-même. Dans les deux cas de figure $(1,4,5$ : schème pronominal primaire vs. 2,3 : schème transitif primaire) on rencontre donc des illustrations convaincantes à côté d'illustrations sujettes à caution.

\section{La structuration sémantique des entrées: les opérateurs et leurs composants primaires}

Pour y voir plus clair, nous disposons d'un outil précieux: contrairement aux tables du lexique-grammaire (dans le domaine verbal: Gross, 1975; Boons, Guillet \& Leclère, 1976; Guillet \& Leclère, 1992), le classement de JD \& FDC ne se fonde pas uniquement sur des propriétés distributionnelles et transformationnelles, mais aussi essentiellement sur 
l'attribution à chaque entrée d'un opérateur décomposable en une chaine de primitives sémantiques que $\mathrm{j}$ 'appellerai des «sous-opérateurs ». L'hypothèse à tester est que la répartition en 8891 schèmes composés à source transitive T.*..-P.... et 1264 schèmes composés à source pronominale P...•-T•..• est pilotée par 1'attribution aux entrées lexicales concernées de sous-opérateurs spécifiques. Dans un premier temps nous allons chercher à identifier les sous-opérateurs qui interviennent de manière récurrente dans les entrées de chacun des deux types de schèmes composés, puis nous dégagerons parmi ces sous-opérateurs la liste de ceux qui contribuent à une interprétation factitive ou non factitive de la construction transitive.

Les sous-opérateurs intervenant dans les entrées classées dans chacun des deux types de schèmes composés

Par ordre décroissant, les 12 sous-opérateurs intervenant en premier (jusqu'à un effectif de 10) dans les 1264 entrées associées au type de schème composé P...•-T $\cdots .$. sont : sent [sentiment], 117; av. som [avoir dans corps], 79; $\mathbf{d}$ [devenir], 51; li [lier], 37; r/d [rendre/devenir], 32; mut [changer], 28; dmut [défaire], 22; lc [être ds lieu], 15; rag [être/mettre en morceaux (rag)], 15; ex [sortir], 13; ger. mens [gérer mentalement], 10 ; ire, [aller], 10.

Les 18 sous-opérateurs intervenant en premier (jusqu'à un effectif de 20) dans les 8891 entrées du type T•..•-P»... sont mun [munir], 526; dmu [démunir], 524; *r/d [rendre/devenir], 510; *rag [mettre en morceaux (rag)], 365; m.e.état [mettre en état], 306; fab [fabriquer], 279; f. ex [faire sortir], $157 ; *$ lc [être ds lieu], $151 ;$ f. mvt [faire mouvement], 102; fac [réaliser], 83 ; scrut [scruter], 80 ; f. ire [faire aller], $65 ; *$ li [lier], 60 ; percep [percevoir], 54 ; grp [grip, saisir], 48; *dmut [défaire], $39 ; *$ mut [changer], 38 ; dgrp [lâcher], 23.

Les six sous-opérateurs mentionnés dans les deux listes sont précédés d'un * : $\{\mathbf{r} / \mathbf{d}, \mathbf{r a g}, \mathbf{l c}, \mathbf{l i}, \mathbf{d m u t}, \mathbf{m u t}\}$. Ils représentent respectivement la moitié et un tiers des sous-opérateurs caractéristiques de chacun des deux types de schèmes composés. 


\section{Les sous-opérateurs indices de (non) factitivité ${ }^{9}$}

Les auteurs distinguent deux types de construction transitive ${ }^{10}$ :

«Conventionnellement, on a étiqueté

- transitif direct $(\mathrm{T})$

deux types de construction, (1) le transitif direct proprement dit, où le groupe verbal est constitué soit d'un complément objet direct, soit de deux compléments, l'un direct et l'autre prépositionnel; par exemple: épauler qn, donner $q c$ à $q n$, promener son chien dans les rues; (2) le factitif, de même forme que le transitif, mais impliquant l'équivalence avec faire suivi d'un intransitif ou d'un pronominal; par exemple le joueur dévie la balle (= fait que la balle dévie), le film a ennuyé Paul ( $=$ a fait que Paul s'est ennuyé) $\gg$ [1997a, p. IX et 1997b, p.60]

Or il est clair que, si certains sous-opérateurs sont neutres à l'égard de la factivité, par exemple mut (changer) qui intervient seul, sans combinaison avec f., dans des expressions factitives comme dans des expression non factitives, $d$ 'autres sous-opérateurs sont en revanche des facteurs respectivement de factitivité et de non factitivité (cf. tableau 2).

Sept sous-opérateurs donnent à l'opérateur composé une valeur explicitement factitive: f. (faire, combiné par exemple à ire : faire aller, ou à sent : faire sentir) et ses trois variantes m. (mettre, combiné par exemple à e. état : mettre en état, ou à e. mvt : mettre en mouvement; fab (fabriquer) et fac (réaliser); grp (saisir); mun (munir) et son inverse dmu (démunir), quand ils ne sont pas suivis de soi.

9. La propriété de factitivité de la construction verbale correspond à la notion de cadre prédicatif causatif dans François (2003).

10. Cette distinction est déjà évoquée par Dubois (1969:35): «la phrase (1) Pierre amuse Paul s'analyse en (2) Pierre fait ceci QUE Paul est amusé». Il est à noter par ailleurs que la factitivité selon JD \& FDC ne correspond que partiellement à l'usage de celles des sémantiques formelles qui pratiquent la décomposition du prédicat verbal factitif (cf. Dowty, 1979 et son application par van Valin \& La Polla, 1997 et van Valin, 2005), car les auteurs ne classent pas les verbes trivalents dans la rubrique «factitif». 
Inversement trois sous-opérateurs constituent un facteur de non-factitivité : av. (avoir, combiné par exemple à som: dans corps) par sa valeur statique, $\mathbf{d}$ (devenir) parce qu'il commute avec r/d (rendre/devenir) et sent (sentir) parce qu'il commute avec f. sent (faire sentir).

\begin{tabular}{|c|c|c|c|}
\hline & $\mathbf{P}-\mathbf{T}$ & $\mathbf{T}-\mathbf{P}$ & Factitif \\
\hline $\begin{array}{l}\text { av. } \\
\text { d } \\
\text { sent }\end{array}$ & $\begin{array}{l}113 \\
21 \\
72\end{array}$ & $\begin{array}{l}2 \\
0 \\
10\end{array}$ & NON \\
\hline f. & 0 & 945 & \\
\hline $\mathbf{m}$ & 0 & 489 & \\
\hline $\mathrm{dmu} \mathbf{u}^{11}$ & & 4 & 782 \\
\hline $\mathrm{fab}$ & 0 & 293 & OUI \\
\hline fac & 0 & 98 & \\
\hline $\operatorname{grp}^{12}$ & 22 & 368 & \\
\hline $\operatorname{mun}^{13}$ & & 2 & 921 \\
\hline Total non factitif & 206 & 12 & \\
\hline Total factitif & 28 & 3896 & \\
\hline Proportion & $88,0 \%$ & $99,7 \%$ & \\
\hline
\end{tabular}

Tableau 2 - L'intervention des sous-opérateurs de factitivité

et de non factitivité dans les entrées associées aux schèmes syntaxiques des deux types

\section{Un bilan contrasté}

L'opposition entre la transitivité factitive (ou transitivité «sémantique » dans la terminologie de J.P. Desclés, 1998) et la transitivité non factitive (ou transitivité «syntaxique» et sans corrélat sémantique selon Desclés) rend effectivement compte de l'orientation de la relation transformationnelle: pour $88 \%$ des entrées comportant dans leur caractérisation sémantique 1'un des 3 sous-opérateurs explicitement non factitifs identifiés, le schème composé retenu est du type P....-

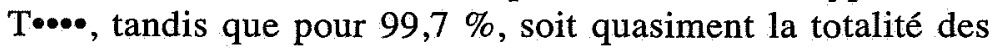

11. En l'absence du sous-opérateur «soi». La paire «dmu soi » fournit 44 entrées dans la combinaison P-T.

12. Dans la combinaison P-T, 22 entrées de consommation d'alcool, de drogue ou d'eau.

13. Dans la combinaison P-T, s'extasier et s'emboliser. La paire «mun soi » fournit 136 entrées dans la combinaison P-T. 
entrées caractérisées sémantiquement par 1'un des 7 sous-opérateurs explicitement factitifs, le schème est du type T..... P.o.•.

Cependant, il reste un problème qui se présente sous deux facettes: d'une part 136 entrées dont l'opérateur comporte inversement la paire «mun soi » [se munir] sont associées à 8 types différents de schèmes composés, tous du type P•...T॰.», par exemple motoriser 02(s) au type [P10b0 T1108], on se motorise $\rightarrow$ la Poste motorise les facteurs; d'autre part 44 entrées caractérisées inversement par la paire de sous-opérateurs «dmu soi» [se démunir] sont également associées à un schème composé du type P.o.•-T....:

(6) [P10b0 T11b0], ex. Marie se débotte $\rightarrow$ Paul débotte Marie

(7) [P30b0 T3300], ex. les finales se désaccentuent $\rightarrow$ l'évolution désaccentue les finales

(8) [P30b0 T33b0], ex, le temps se dégage $\rightarrow$ la marée dégage le temps

(9) [P90b0 T31b0], ex. Paul se déplume $\rightarrow$ l'âge déplume Paul,

(10) [P90b0 T3900], ex. la peau de Marie se dépigmente $\rightarrow$ La maladie dépigmente la peau de Marie.

Ce qui frappe dans les exemples (6-10) appartenant à 5 classes différentes, c'est leur disparité en termes de contrôle du procès: dans le type [P10b0 T11b0] (28 entrées), le référent humain du sujet de la construction pronominale contrôle bien l'action et la paire «dmu + soi » est pertinente ${ }^{14}$, en revanche dans les quatre autres cas (14 entrées), le référent du sujet n'est pas un humain ou bien c'est un humain qui n'a pas de pouvoir de contrôle sur l'évènement (se déplumer), ce qui devrait être caractérisé sémantiquement par «cesser d'avoir» et non par «se démunir». Inversement c'est pour ces

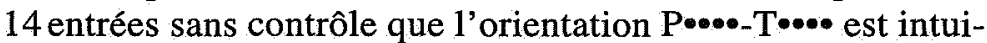
tivement admissible, car, présenté indépendamment de toute instance causatrice, l'évènement incontrôlé peut être considéré comme primaire par rapport au même évènement mis en relation avec une telle instance ${ }^{15}$ (la marée influençant le

14. Pour toutes les entrées du type, le sujet de la construction pronominale est toujours un humain qui contrôle l'action: se déchausser, se décoiffer, se déshabiller, se dévêtir, etc.

15. Cf. François (1998) sur le «cognitive packaging» et le «grammatical packaging 》 de l'évènement en relation ou sans relation avec une instance causatrice. Cette terminologie est empruntée à Givón (1991). 
temps, l'évolution déterminant le changement phonétique, l'âge altérant l'aspect physique de Paul, la maladie altérant la peau de Marie).

Ma contre-proposition consiste donc en l'occurrence à inverser en [T11b0 P10b0] le schème composé [P10b0 $\mathrm{T} 11 \mathrm{~b} 0$ ] des 28 entrées exprimant 1'action d'enlever un de ses propres vêtements (ou assimilés) mais inversement de le conserver pour les quatre autres classes. Le même raisonnement vaut pour les 88 entrées sur 132 caractérisées par «mun

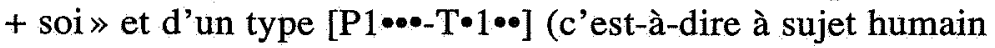
de la construction pronominale et objet humain de la construction transitive) pour lesquelles l'inversion du schème composé en $\left[\mathrm{T} \bullet 1 \bullet-\mathrm{P} 1 \cdots \cdot{ }^{\circ}\right.$ est à recommander pour aboutir à une corrélation pertinente entre opérateurs et schèmes composés. Les modifications proposées sont:

$\rightarrow[\mathrm{P} 10 \mathrm{~b} 0$ T11b0] à remplacer par [T11b0 P10b0] pour les 28 entrées à opérateur «dmu soi $\mathrm{D}<$ vêtement $>»$ :

débâcher 03(s); débotter (s); débraguetter (s); déceindre (s); déchausser $01(\mathrm{~s})$; décoiffer 01(s); décolleter 01(s); décravater (s); décuirasser (s) ; déculotter 01(s); défringuer (s); défrusquer (s); défrusquiner (s); déganter $(\mathrm{s})$; déharnacher 02(s); déloquer (s); démasquer 01(s); dépapilloter (s); dépenailler (ê); dépoitrailler (ê) ; désendimancher (s); désenturbanner (s); déshabiller 01(s); déshabiller 05(s); dévêtir (s) ; effeuiller 02(s); redéchausser (s); redéculotter (s);

$\rightarrow[\mathrm{P} 1 \bullet \bullet-\mathrm{T} \bullet 1 \bullet \bullet]$ à remplacer par $[\mathrm{T} \bullet 1 \bullet \bullet-\mathrm{P} 1 \bullet \bullet \cdot]$ pour les 88 entrées à opérateur «mun soi $\mathrm{D}<$ vêtement/substance $>$ »:

absinther (s); alcooliser 03(s); armer 09(s); autoéquiper (s); automédiquer (s) ; aviner 02(s); bâcher 03(s) ; bâcher 04(s); béquiller 04(s); beurrer 03(s); blinder 06(s); boissonner (s); botter 01(s); bourrer $07(\mathrm{~s})$; braguetter (s); camer (s); camisoler (s); camphrer 02(s); capuchonner $01(\mathrm{~s})$; casquer 01(s); ceinturer 03(s); changer 18(s); chapeauter 01(s); charger $15(\mathrm{~s})$; charger $19(\mathrm{~s})$; chausser $01(\mathrm{~s})$; coiffer $03(\mathrm{~s})$; corseter $01(\mathrm{~s})$; costumer (s); couvrir $24(\mathrm{~s})$; cravater $01(\mathrm{~s})$; culotter 01(s) ; dégueniller (ê); délecter 01(s); déloqueter (ê); droguer 03(s); embéguiner (s) ; emmanteler (s); emmasquer (s); empanacher (s); emperlouser (s); emperruquer (s); encagouler (s); encapuchonner 01(s); encorneter (s); endimancher (s); engueniller (s); enjuponner 01(s); ensoutaner (s); enturbanner (s); farder 01(s); fringuer (s); froquer (s) ; frusquer (s) ; ganteler $(\mathrm{s})$; ganter $01(\mathrm{~s})$; ganter $03(\mathrm{~s})$; gargariser $03(\mathrm{~s})$; gileter $(\mathrm{s})$; guêtrer (s); habiller 02(s); harnacher 03(s); linger (s); loquer (s); lunetter (s); masquer 01(s); motoriser 02(s); munir 03(s); munir 04(s); narcotiser 02(s) ; nipper (s) ; papilloter 04(s) ; pistacher (s) ; piver (s) ; poisser 04(s); poivrer 03(s); poudrer 04(s); resservir 05(s); restaurer 05(s); rhabiller 01(s); sabouler 02(s); schnoufer (s); schnouffer (s); servir 16(s); smacker (s); speeder (s); sustenter 02(s); torcher 07(s). 


\section{RÉFÉRENCES}

Boons J.P., Guillet A. \& LeClère CH. (1976): La structure des phrases simples en français: Constructions intransitives, Genève-Paris, Droz.

Desclés, J.-P. (1998): Transitivité sémantique, transitivité syntaxique, in A. Rousseau (dir. 1998), La transitivité, Villeneuve d'Ascq, Presses du Septentrion, 161-180.

DowTy, D. (1979): Word meaning and Montague Grammar, Dordrecht, Kluwer.

DUBoIs, J. (1969) : Grammaire structurale du français III : La phrase et les transformations, Paris, Larousse.

DUBOIS, J. \& DUBOIS-CHARLIER, F. (1997a): Les verbes français, Paris, Larousse.

Dubois, J. \& Dubois-Charlier, F. (1997b) : Synonymie syntaxique et classsification des verbes français, Langages, $128,51-71$.

FrançoIs J. (1998): Théorie multifactorielle de la transitivité, 'différentiel de participation' et classes aspectuelles et actancielles de prédication, in A. Rousseau (dir.), La transitivité, Lille, Presses du Septentrion, 181-201.

FrançoIs, J. (2003) : La prédication verbale et les cadres prédicatifs, Louvain, Peeters [Bibliothèque de 1'Information grammaticale 54].

François, J., Le Pesant, D. \& Leeman, D. (2007) : Présentation de la classification des Verbes français de Jean Dubois et Françoise Dubois-Charlier, in J. François, D. Le Pesant \& D. Leeman (dir. 2007).

Françors, J., Le Pesant, D. \& Leeman, D. (dir. 2007) : Les classes syntactico-sémantiques des verbes français, Langue française, 153.

Grvón, T. (1991): Serial verbs and the mental reality of 'Event' : Grammatical vs cognitive packaging, in B. Heine \& E. Traugott (eds.), Approaches to Grammaticalization, vol.1., Amsterdam, Benjamins, 81-127.

Gross, M. (1975): Méthodes en syntaxe. Régime des constructions complétives, Paris, Hermann.

GUILlET, A. \& LeCLÈRE, Ch. (1992): La structure des phrases simples en français 2 : Constructions transitives locatives, Genève-Paris, Droz. 
PeETers, B (ed. 2006): Semantic primes and universal grammar - Empirical evidence from the Romance languages, Amsterdam et al., Benjamins.

RUWET, N. (1972) : Les constructions pronominales neutres et moyennes, in N. Ruwet (dir.), Théorie syntaxique et syntaxe du français, Paris, Seuil, 87-125.

TESNière, L. (1969): Éléments de syntaxe structurale, Paris, Klincksieck.

VAN VALIN, R. (2005): Exploring the Syntax-Semantics Interface, Cambridge, Cambridge University Press.

VAN VAlin, R. \& LAPolla, R. (1997): Syntax - Structure, Function, Meaning, Cambridge, Cambridge University Press. 